

P. Kern, General multiparameter version of Gnedenko's transfer theorem, Теория вероятн. и ее примен., 2015, том 60, выпуск $1,198-206$

DOI: https://doi.org/10.4213/tvp4615

Использование Общероссийского математического портала Math-Net.Ru подразумевает, что вы прочитали и согласны с пользовательским соглашением http://www . mathnet.ru/rus/agreement

Параметры загрузки:

IP : 54.92 .164 .108

26 апреля 2023 г., 17:57:54

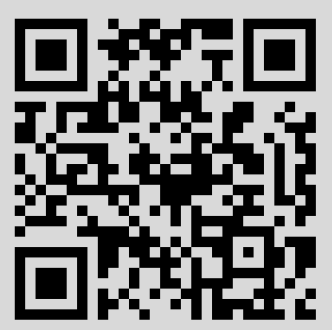


5. Azaïs J.-M., Wschebor M. Level Sets and Extrema of Random Processes and Fields. Hoboken: Wiley, 2009, 393 p.

6. Berman S.M. Local times and sample functions properties of stationary Gaussian processes. - Trans. Amer. Math. Soc., 1969, v. 137, p. 277-299.

7. Biermé H., Desolneux A. Crossings of smooth shot noise processes. - Ann. Appl. Probab., 2012, v. 22, № 6, p. 2240-2281.

8. Bulinski A. Central limit theorem for random fields and applications. - Advances in Data Analysis. Boston: Birkhäuser, 2010, p. 141-150.

9. Bulinski A., Suquet Ch. Normal approximation for quasi-associated random fields. Statist. Probab. Lett., 2001, v. 54, № 2, p. 215-226.

10. Iribarren I. Asymptotic behaviour of the integral of a function on the level set of a mixing random field. - Probab. Math. Statist., 1989, v. 10, № 1, p. 45-56.

11. Kratz M. Level crossings and other level functionals of stationary Gaussian processes. - Probab. Surv., 2006, v. 3, p. 230-288.

12. Shashkin A. A functional central limit theorem for the level measure of a Gaussian random field. - Statist. Probab. Lett., 2013, v. 83, № 2, p. 637-643.

Поступила в редакцию 16.X.2013

Исправленный вариант

27.III.2014

(c) 2015 г.

KERN P.*

\title{
A GENERAL MULTIPARAMETER VERSION OF GNEDENKO'S TRANSFER THEOREM
}

\begin{abstract}
Предельные теоремы для случайного числа независимых случайных величин часто называют теоремами о переносе. Начало исследованиям в этом направлении для сумм случайных величин с независимым случайным объемом выборки было положено Б. В. Гнеденко. Мы приводим допускающую широкое применение теорему о переносе для случайных величин на общем метрическом пространстве со случайными многомерными параметрами вместо случайных объемов выборки. Это обобщает существенный принцип, лежащий в основе известных в литературе результатов типа теорем о переносе.
\end{abstract}

Ключевые слова и фразы: теорема о переносе, случайная остановка, случайная замена времени, независимые случайные индексы, случайные поля, отрицательная ассоциированность, инфинитезимальная схема серий, полуустойчивая область притяжения, случайные размещения.

1. Introduction. In 1969 Gnedenko and Fahim [15] proved the following limit theorem for sums of a random number of real-valued random variables; see also [14] for the precise assumptions. For every $n \in \mathbb{N}$ let $\left(X_{n, k}\right)_{k \in \mathbb{N}}$ be a sequence of independent and identically distributed (i.i.d.) random variables, and let $\left(T_{n}\right)_{n \in \mathbb{N}}$ be a sequence of positive integer-valued random variables such that $T_{n}$ and $\left(X_{n, k}\right)_{k \in \mathbb{N}}$ are independent for every $n \in \mathbb{N}$. If there exists a sequence $k_{n} \rightarrow \infty$ such that as $n \rightarrow \infty$

$$
\sum_{k=1}^{k_{n}} X_{n, k} \Rightarrow \mu \quad \text { and } \quad \frac{T_{n}}{k_{n}} \Rightarrow \rho
$$

* Mathematisches Institut, Heinrich-Heine-Universität Düsseldorf, 
for some probability distributions $\mu$ on $\mathbb{R}$, respectively, $\rho$ on $(0, \infty)$, then the random sum converges to a mixture distribution

$$
\sum_{k=1}^{T_{n}} X_{n, k} \Rightarrow \int_{0}^{\infty} \mu^{t} \mathrm{~d} \rho(t),
$$

where « $\Rightarrow \gg$ denotes convergence in distribution, i.e., the distribution of the random variable on the left-hand side converges weakly to the measure on the right-hand side, and $\mu^{t}$ denotes the $t$-fold convolution power of the necessarily infinitely divisible distribution $\mu$, well defined by the Lévy-Khinchin formula. The result is frequently called the transfer theorem of Gnedenko, since randomization of the sample size transfers the limits in (1.1) to a randomized limit in (1.2). There is an ongoing considerable interest in random limit theorems of this kind from numerous applications, and today there exists a vast literature on transfer theorems generalizing the above. Without demanding to give a complete list, we refer to [31], [34], [27], [13], [25], [26], [11] and the literature cited therein, to mention just a few direct generalizations. Comprehensive studies of random limit theorems of various kind are given in the monographs [17], [16], [7], [33]. Although there is an intrinsic principle behind all the transfer type results, a new proof is given in every new situation. Our aim is to capture this principle and to prove a widely applicable version of the transfer theorem for randomly indexed sequences of random elements on a general metric space. For this reason we replace the random number $T_{n}$ by a random multiparameter in $\mathbb{N}^{d}$. We will introduce a natural homeomorphism on the discrete multiparameters that serves as a control mapping for the transfer mechanism. We also aim to consider general state spaces for the random variables $X_{n, k}$. Since we are concerned with weak convergence of probability measures, a requirement on our state space $E$ is that a probability measure $\mu$ on $E$ is uniquely determined by the values $\int_{E} f d \mu$ for bounded and continuous functions $f: E \rightarrow \mathbb{R}$, i.e., the Riesz theorem applies. Thus we may consider $E$ to be a general metric space; see [30], [20] for details. In Section 2 we present our general transfer theorem and briefly show how it covers classical transfer results appearing in the literature. In Section 3 we focus on three specific examples that give a glance on possible applications of our general transfer theorem.

2. Main result. Let

$$
\mathscr{F}=\left\{Y_{\mathbf{n}}: \mathbf{n}=\left(n_{1}, \ldots, n_{d}\right) \in \mathbb{N}^{d}\right\}
$$

be a family of $E$-valued random variables, where $E$ is a metric space equipped with its Borel $\sigma$-algebra. We use boldface notation for multiparameters. Let $\phi: \mathbb{N}^{d} \rightarrow D \subseteq \mathbb{R}^{\ell}$ be a homeomorphism. Note that we consider $\mathbb{N}^{d}$ and $D$ as subsets of $\mathbb{R}^{d}$ and $\mathbb{R}^{\ell}$, respectively, with the induced standard topology. Hence any subset of $\mathbb{N}^{d}$ is a Borel set and any mapping $\phi: \mathbb{N}^{d} \rightarrow \mathbb{R}^{\ell}$ is continuous. The only assumptions we have to make to ensure that $\phi$ is a homeomorphism are that $\phi$ is injective and that the image $D=\operatorname{Im} \phi$ is discrete in the sense that intersections of $D$ with sufficiently small open neighborhoods of any $\mathbf{x} \in D$ coincide with the single point set $\{\mathbf{x}\}$. Now let $\Delta \subseteq \bar{D} \backslash D$ be a nonempty Borel set of limit points of $D$, where $\bar{D}$ denotes the closure of $D$ in $\mathbb{R}^{\ell}$. We assume that for any $\mathbf{t}=\left(t_{1}, \ldots, t_{\ell}\right) \in \Delta$ and any sequence $\left(\mathbf{N}_{n}\right)_{n \in \mathbb{N}}$ in $\mathbb{N}^{d}$ we have

$$
Y_{\mathbf{N}_{n}} \Rightarrow \mu_{\mathbf{t}} \quad \text { whenever } \quad \phi\left(\mathbf{N}_{n}\right) \rightarrow \mathbf{t},
$$

where $\left\{\mu_{\mathbf{t}}: \mathbf{t} \in \Delta\right\}$ is a weakly continuous family of probability measures on $E$. Thus the homeomorphism $\phi$ serves as a control mapping for weak convergence in (2.1). For randomizations of the multiparameter $\mathbf{n}$ of $\mathscr{F}$ we are able to give the following general version of a transfer theorem.

Theorem 1. With the above assumptions and notation let $\left(\mathbf{T}_{n}\right)_{n \in \mathbb{N}}$ be a sequence of random variables with values in $\mathbb{N}^{d}$ such that $\mathbf{T}_{n}$ and $\mathscr{F}$ are independent for every $n \in \mathbb{N}$. 
Suppose that $\phi\left(\mathbf{T}_{n}\right) \Rightarrow \rho$ for some probability distribution $\rho$ with $\rho(\Delta)=1$. Then we have as $n \rightarrow \infty$

$$
Y_{\mathbf{T}_{n}} \Rightarrow \int_{\Delta} \mu_{\mathbf{t}} \mathrm{d} \rho(\mathbf{t}) .
$$

$\mathrm{P} \mathrm{r}$ o o f. For a probability measure $\mu$ and a fixed, bounded, and continuous function $f: E \rightarrow \mathbb{R}$ we use the abbreviation

$$
\langle\mu, f\rangle=\int_{E} f(x) \mathrm{d} \mu(x) .
$$

Let $\nu(\mathbf{n})=P_{Y_{\mathbf{n}}}$ denote the distribution of $Y_{\mathbf{n}}$, then $\varphi: D \rightarrow \mathbb{R}$ defined by

$$
\varphi(\mathbf{t})=\left\langle\nu\left(\phi^{-1}(\mathbf{t})\right), f\right\rangle \quad \text { for } \mathbf{t} \in D
$$

is a bounded continuous function, where continuity is due to the fact that $D$ is discrete. We will now extend $\varphi$ to a bounded continuous function $\psi: D \cup \Delta \rightarrow \mathbb{R}$ by

$$
\psi(\mathbf{t})= \begin{cases}\varphi(\mathbf{t}) & \text { if } \mathbf{t} \in D \\ \left\langle\mu_{\mathbf{t}}, f\right\rangle & \text { if } \mathbf{t} \in \Delta\end{cases}
$$

Clearly, $\psi$ is bounded on $D \cup \Delta$, and continuous on $D$ and on $\Delta$. The latter is due to our assumption on weak continuity of $\mathbf{t} \mapsto \mu_{\mathbf{t}}$ on $\Delta$. Now let $\mathbf{t} \in \Delta$ be arbitrary and let $\left(\mathbf{t}_{n}\right)_{n \in \mathbb{N}}$ be a sequence in $D$ such that $\mathbf{t}_{n} \rightarrow \mathbf{t}$. Then $\mathbf{N}_{n}=\phi^{-1}\left(\mathbf{t}_{n}\right)$ fulfills the right-hand side of (2.1) and thus we get

$$
\psi\left(\mathbf{t}_{n}\right)=\varphi\left(\mathbf{t}_{n}\right)=\left\langle\nu\left(\phi^{-1}\left(\mathbf{t}_{n}\right)\right), f\right\rangle=\left\langle\nu\left(\mathbf{N}_{n}\right), f\right\rangle \rightarrow\left\langle\mu_{\mathbf{t}}, f\right\rangle=\psi(\mathbf{t})
$$

showing continuity of $\psi$.

By the independence assumption together with $\phi\left(\mathbf{T}_{n}\right) \Rightarrow \rho$ we finally obtain

$$
\begin{aligned}
\left\langle P_{Y_{\mathbf{T}_{n}}}, f\right\rangle & =\int_{\mathbb{N}^{d}}\langle\nu(\mathbf{n}), f\rangle \mathrm{d} P_{\mathbf{T}_{n}}(\mathbf{n})=\int_{D}\left\langle\nu\left(\phi^{-1}(\mathbf{t})\right), f\right\rangle \mathrm{d} P_{\phi\left(\mathbf{T}_{n}\right)}(\mathbf{t}) \\
& =\int_{D \cup \Delta} \psi(\mathbf{t}) \mathrm{d} P_{\phi\left(\mathbf{T}_{n}\right)}(\mathbf{t}) \rightarrow \int_{D \cup \Delta} \psi(\mathbf{t}) \mathrm{d} \rho(\mathbf{t}) \\
& =\int_{\Delta}\left\langle\mu_{\mathbf{t}}, f\right\rangle \mathrm{d} \rho(\mathbf{t})=\left\langle\int_{\Delta} \mu_{\mathbf{t}} \mathrm{d} \rho(\mathbf{t}), f\right\rangle .
\end{aligned}
$$

This proves our assertion, since the bounded continuous function $f$ is arbitrary. Theorem 1 is proved.

We will now apply Theorem 1 to the classical convergence scheme of row-sums of triangular arrays of random variables, extending versions of the transfer theorem on second countable locally compact groups by Hazod [18], [19] and on separable Banach spaces by Siegel [32]. We have to assume second countability to ensure that products of Borel measurable random variables are again Borel measurable; cf. [12, Proposition 4.1.7]. For a metric space $E$ second countability is equivalent to separability; cf. [12, Proposition 2.1.4]. Our multiparameter setting allows us to extend considerations to random fields as follows. For notational convenience we will write the group multiplication as summation. Note that in case of non-Abelian groups this operation has to be taken in a certain fixed order. In the sequel, relations and operations on multiparameters in $\mathbb{R}^{d}$ are always meant componentwise.

Let $E$ be a separable metrizable group. For $n \in \mathbb{N}$, let $\mathscr{F}_{n}=\left\{X_{n, \mathbf{k}}: \mathbf{k} \in \mathbb{N}^{d}\right\}$ be a random field, i.e., a family of $E$-valued random variables, and define for any $n \in \mathbb{N}$ and $\mathbf{N} \in \mathbb{N}^{d}$

$$
Y_{n, \mathbf{N}}=\sum_{\mathbf{k} \leqslant \mathbf{N}} X_{n, \mathbf{k}}
$$

Suppose the existence of a (componentwise) strictly increasing sequence $\left(\mathbf{k}_{n}\right)_{n \in \mathbb{N}}$ in $\mathbb{N}^{d}$ such that for some $\varnothing \neq T \subseteq[0, \infty)^{d}$ and some family $\left\{\mu_{\mathbf{t}}: \mathbf{t} \in T\right\}$ of probability distributions on $E$ we have as $n \rightarrow \infty$

$$
Y_{n,\left\lfloor\mathbf{k}_{n} \mathbf{t}\right\rfloor} \Rightarrow \mu_{\mathbf{t}},
$$


uniformly on compact subsets of $\mathbf{t} \in T$, i.e., for an arbitrary sequence $\left(\mathbf{t}_{n}\right)_{n \in \mathbb{N}}$ in $T$ with

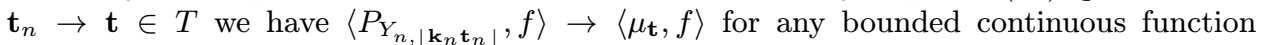
$f: E \rightarrow \mathbb{R}$.

Corollary 1. With the above assumptions and notation let $\left(\mathbf{T}_{n}\right)_{n \in \mathbb{N}}$ be a sequence of random variables with values in $\mathbb{N}^{d}$ such that $\mathbf{T}_{n}$ and $\mathscr{F}_{n}$ are independent for every $n \in \mathbb{N}$. Suppose $\mathbf{T}_{n} / \mathbf{k}_{n} \Rightarrow \rho$ for some probability distribution $\rho$ with $\rho(T)=1$. Then we have as $n \rightarrow \infty$

$$
Y_{n, \mathbf{T}_{n}} \Rightarrow \int_{T} \mu_{\mathbf{t}} \mathrm{d} \rho(\mathbf{t}) .
$$

P r o o f. Let $\mathscr{F}=\left\{Y_{n, \mathbf{N}}: n \in \mathbb{N}, \mathbf{N} \in \mathbb{N}^{d}\right\}$ and note that without loss of generality we may assume that $\mathbf{T}_{n}$ and $\mathscr{F}$ are independent for every $n \in \mathbb{N}$. Otherwise we may change to random variables $\mathbf{T}_{n}^{\prime}$ independent of $\mathscr{F}$ and with the same distribution as $\mathbf{T}_{n}$ which leave the assertion in (2.4) unchanged. One may easily check that $\phi: \mathbb{N}^{1+d} \rightarrow D$ defined by $\phi(n, \mathbf{N})=\left(1 / n, \mathbf{N} / \mathbf{k}_{n}\right)$ with $D=\operatorname{Im} \phi$ is a homeomorphism. For $\Delta=\{0\} \times T \subseteq \bar{D} \backslash D$ and any sequence $\left(n_{k}, \mathbf{N}_{k}\right) \in \mathbb{N}^{1+d}$ with $\phi\left(n_{k}, \mathbf{N}_{k}\right) \rightarrow(0, \mathbf{t}) \in \Delta$ we have $n_{k} \rightarrow \infty$ and $\mathbf{m}_{k}=\mathbf{N}_{k} / \mathbf{k}_{n_{k}} \rightarrow \mathbf{t}$ so that by (2.3) we have

$$
Y_{n_{k}, \mathbf{N}_{k}}=Y_{n_{k},\left\lfloor\mathbf{k}_{n_{k}} \mathbf{m}_{k}\right\rfloor} \Rightarrow \mu_{\mathbf{t}} .
$$

Note that the family $\left\{\mu_{0, \mathbf{t}}=\mu_{\mathbf{t}}:(0, \mathbf{t}) \in \Delta\right\}$ is weakly continuous due to the uniform compact convergence in (2.3). Hence (2.1) is fulfilled and we further have $\phi\left(n, \mathbf{T}_{n}\right)=$ $\left(1 / n, \mathbf{T}_{n} / \mathbf{k}_{n}\right) \Rightarrow \delta_{0} \otimes \rho$. An application of Theorem 1 now gives

$$
Y_{n, \mathbf{T}_{n}} \Rightarrow \int_{\Delta} \mu_{0, \mathbf{t}} \mathrm{d}\left(\delta_{0} \otimes \rho\right)(s, \mathbf{t})=\int_{T} \mu_{\mathbf{t}} \mathrm{d} \rho(\mathbf{t})
$$

concluding the proof of Corollary 1.

In case $d=1$ and $E=\mathbb{R}^{m}$ the classical transfer theorem of Gnedenko [14], [15] is a special case of Corollary 1 as follows. Suppose $\left(X_{n, k}\right)_{k \in \mathbb{N}}$ is a sequence of i.i.d. random variables for every $n \in \mathbb{N}$ such that for some strictly increasing sequence $\left(k_{n}\right)_{n \in \mathbb{N}}$ in $\mathbb{N}$ we have

$$
Y_{n, k_{n}}=\sum_{k=1}^{k_{n}} X_{n, k} \Rightarrow \mu,
$$

where $\mu$ is a necessarily infinitely divisible probability distribution on $\mathbb{R}^{m}$. Then $(2.3)$ is fulfilled, where $\left(\mu_{t}\right)_{t \geqslant 0}$ is the continuous convolution semigroup generated by $\mu$. Thus in this situation Corollary 1 restates the transfer theorem of Gnedenko as presented in Introduction. Note that the proof in [14], [15] is given in terms of convergence of Fourier transforms. Further note that instead of the above partial sums it is also possible to consider partial (componentwise) maxima of random vectors [8], [1], [2] or partial products of random variables [28].

$\mathrm{R}$ e $\mathrm{m}$ a $\mathrm{r} \mathrm{k}$ 1. Our random fields approach in Corollary 1 can be further applied to the convergence of finite dimensional marginal distributions of certain randomly stopped partial sum processes on $E$ (e.g., [6, Theorem 4.1] for $E=\mathbb{R}^{m}$ ). It is also possible to apply Theorem 1 to obtain a transfer type result for randomly stopped stochastic processes on the path space $E=D([0, \infty), S)$ of càdlàg functions on a complete separable metric space $S$, since $D([0, \infty), S)$ can itself be seen as a complete separable metric space; see [9], [23] for details.

3. Examples. To illustrate some possible applications of our Theorem 1 we aim to give further examples in which different limits under different regimes arise with a mixture distribution of the transfer type limit.

3.1. A transfer theorem for negatively associated random fields. Our first example is a generalization of Gnedenko's transfer theorem for partial sums in a multiparameter and non-i.i.d. setting. Let $\left\{X_{\mathbf{n}}: \mathbf{n} \in \mathbb{N}^{d}\right\}$ be a field of stationary, centered and 
negatively associated random variables with positive and finite second moment. Stationarity means that $\left\{X_{\mathbf{n}+\mathbf{m}}: \mathbf{n} \in \mathbb{N}^{d}\right\}$ is distributed as $\left\{X_{\mathbf{n}}: \mathbf{n} \in \mathbb{N}^{d}\right\}$ for every $\mathbf{m} \in \mathbb{N}^{d}$. Negative association was introduced in [22] and claims that

$$
\operatorname{Cov}\left(f\left(X_{\mathbf{n}}: \mathbf{n} \in I\right), g\left(X_{\mathbf{m}}: \mathbf{m} \in J\right)\right) \leqslant 0
$$

for every pair $I, J$ of disjoint subsets of $\mathbb{N}^{d}$ and any coordinatewise increasing functions $f, g$ with $\mathbb{E}\left[f^{2}\left(X_{\mathbf{n}}: \mathbf{n} \in I\right)\right]<\infty$ and $\mathbb{E}\left[g^{2}\left(X_{\mathbf{m}}: \mathbf{m} \in J\right)\right]<\infty$. Let us define

$$
Y_{\mathbf{n}, \mathbf{N}}=\frac{1}{\sqrt{|\mathbf{n}|}} \sum_{\mathbf{k} \leqslant \mathbf{N}} X_{\mathbf{k}}
$$

for $\mathbf{n}, \mathbf{N} \in \mathbb{N}^{d}$, where $|\mathbf{n}|=\prod_{i=1}^{d} n_{i}$. Introduce a homeomorphism $\phi: \mathbb{N}^{2 d} \rightarrow D$ by

$$
\phi(\mathbf{n}, \mathbf{N})=\left(\frac{1}{\mathbf{n}}, \frac{\mathbf{N}}{\mathbf{n}}\right)
$$

with $D=\operatorname{Im} \phi \subseteq(0, \infty)^{2 d}$. Let $\Delta=\{\mathbf{0}\} \times[0, \infty)^{d} \subseteq \bar{D} \backslash D$, then for any sequence $\left(\mathbf{n}_{k}, \mathbf{N}_{k}\right) \in \mathbb{N}^{2 d}$ with $\phi\left(\mathbf{n}_{k}, \mathbf{N}_{k}\right) \rightarrow(\mathbf{0}, \mathbf{t}) \in \Delta$ we necessarily have $\mathbf{n}_{k} \rightarrow \infty$ (componentwise) and $\mathbf{m}_{k}=\mathbf{N}_{k} / \mathbf{n}_{k} \rightarrow \mathbf{t}$. It follows from Theorem 1 in [35] that

$$
Y_{\mathbf{n}_{k}, \mathbf{N}_{k}}=\frac{1}{\sqrt{\left|\mathbf{n}_{k}\right|}} \sum_{\mathbf{m} \leqslant\left\lfloor\mathbf{n}_{k} \mathbf{m}_{k}\right\rfloor} X_{\mathbf{m}} \Rightarrow \mu_{\mathbf{t}}
$$

where $\mu_{\mathbf{t}}=\mathscr{N}_{0, \sigma^{2}|\mathbf{t}|}$ has a Gaussian distribution for some $\sigma^{2}>0$ depending on the covariance structure of the random field $\left\{X_{\mathbf{n}}: \mathbf{n} \in \mathbb{N}^{d}\right\}$. Clearly, the family of probability distributions $\left\{\mu_{\mathbf{0}, \mathbf{t}}=\mu_{\mathbf{t}}:(\mathbf{0}, \mathbf{t}) \in \Delta\right\}$ is weakly continuous. An application of our Theorem 1 directly leads to the following result, the same way we have proven Corollary 2 .

Corollary 2. Let $\left(\mathbf{T}_{k}\right)_{k \in \mathbb{N}}$ be a sequence of $\mathbb{N}^{d}$-valued random variables such that $\mathbf{T}_{k} / \mathbf{n}_{k} \Rightarrow \rho$ for some sequence $\mathbf{n}_{k} \rightarrow \infty$ in $\mathbb{N}^{d}$ and some probability distribution $\rho$ on $[0, \infty)^{d}$. Then we have

$$
\frac{1}{\sqrt{\left|\mathbf{n}_{k}\right|}} \sum_{\mathbf{m} \leqslant\left\lfloor\mathbf{n}_{k} \mathbf{T}_{k}\right\rfloor} X_{\mathbf{m}} \Rightarrow \int_{[0, \infty)^{d}} \mathscr{N}_{0, \sigma^{2}|\mathbf{t}|} \mathrm{d} \rho(\mathbf{t}) .
$$

3.2. A transfer theorem for semistable domains of attraction. In case $d=1$ and $E=\mathbb{R}^{m}$ we consider the special case of $X_{n, k}=A_{n}\left(X_{k}-a_{n}\right)$ for a sequence $\left(X_{k}\right)_{k \in \mathbb{N}}$ of i.i.d. random vectors on $\mathbb{R}^{m}$, invertible linear operators $A_{n} \in \mathrm{GL}\left(\mathbb{R}^{m}\right)$ and shifts $a_{n} \in \mathbb{R}^{m}$. Suppose there exists a strictly increasing sequence $k_{n} \uparrow \infty$ in $\mathbb{N}$ such that $k_{n+1} / k_{n} \rightarrow c \geqslant 1$

$$
A_{n} \sum_{k=1}^{k_{n}}\left(X_{k}-a_{n}\right) \Rightarrow \mu
$$

for some full probability distribution $\mu$ on $\mathbb{R}^{m}$, where full means not supported on any proper hyperplane of $\mathbb{R}^{m}$. Then $\mu$ is infinitely divisible (in particular it is operator semistable) and it is known that the normalizing operators $A_{n}$ can be chosen such that there exist $E \in \mathrm{GL}\left(\mathbb{R}^{m}\right)$ and $B_{n} \in \mathrm{GL}\left(\mathbb{R}^{m}\right)$ with $B_{k_{n}}=A_{n}$ fulfilling

$$
B_{\lfloor\lambda n\rfloor} B_{n}^{-1} \rightarrow \lambda^{-E}=\sum_{k=0}^{\infty} \frac{(-\ln \lambda)^{k}}{k !} E^{k}
$$

uniformly on compact subsets of $\{\lambda>0\}$. Hence the sequence $\left(B_{n}\right)_{n \in \mathbb{N}}$ is regularly varying with exponent $-E$, see [29] for the details. The case $c=1$ corresponds to operator stability $\mu^{t}=t^{E} \mu * \delta_{a(t)}$ for all $t>0$ and some continuous function $t \mapsto a(t)$. In case $c>1$ operator semistability is given by

$$
\mu^{c}=c^{E} \mu * \delta_{a} \quad \text { for some } a \in \mathbb{R}^{m}
$$


see [29] for details. Moreover, the shifts $\left(a_{n}\right)_{n \in \mathbb{N}}$ can be chosen to be embeddable into a sequence $\left(b_{n}\right)_{n \in \mathbb{N}} \subseteq \mathbb{R}^{m}$ with $b_{k_{n}}=a_{n}$ such that $B_{n} \sum_{k=1}^{n}\left(X_{k}-b_{n}\right)$ is weakly relatively compact with weak limit points

$$
\left\{\mu_{t}=t^{-E}\left(\mu^{t} * \delta_{-a(t)}\right): t \in[1, c)\right\}
$$

where $t \mapsto a(t)$ is continuous with $a(1)=0$ and $a(t) \rightarrow a$ as $t \uparrow c$. Especially, $\mu_{1}=\mu$ and $\mu_{t} \rightarrow \mu=: \mu_{c}$ weakly as $t \uparrow c$ by (3.2). A limit $\mu_{t}$ arises for subsequences of the form $n=k_{p_{n}} t_{n}$ with $t_{n} \rightarrow t \in[1, c]$, where $p_{n} \in \mathbb{N}$ is given by $k_{p_{n}} \leqslant n<k_{p_{n}+1}$; see [3, Section 4] for details. An univariate version $(m=1)$ of the above results is given in [10], where centering shifts are constructed via the quantile function.

Although in general $B_{n} \sum_{k=1}^{n}\left(X_{k}-b_{n}\right)$ does not converge in distribution, it is possible to achieve a limit distribution for random sums by a transfer type theorem as shown in [5]. For $n \in \mathbb{N}$ and the increasing sampling sequence $\left(k_{n}\right)_{n \in \mathbb{N}}$ define $k_{0}=1$ and

$$
\psi(n)=t_{n} \quad \text { if } n=k_{p_{n}} t_{n} \text { with } p_{n} \in \mathbb{N}_{0} \text { given by } k_{p_{n}} \leqslant n<k_{p_{n}+1} .
$$

Corollary 3. Let $\left(T_{n}\right)_{n \in \mathbb{N}}$ be a sequence of positive integer-valued random variables independent of $\left(X_{k}\right)_{k \in \mathbb{N}}$ and assume that $\psi\left(T_{n}\right) \Rightarrow \rho$ for some probability distribution $\rho$ on $[1, c]$. Then we have

$$
B_{T_{n}} \sum_{k=1}^{T_{n}}\left(X_{k}-b_{T_{n}}\right) \Rightarrow \int_{1}^{c} \mu_{t} \mathrm{~d} \rho(t)=\int_{1}^{c} t^{-E}\left(\mu^{t} * \delta_{-a(t)}\right) \mathrm{d} \rho(t) .
$$

P r o o f. Let $\mathscr{F}=\left\{Y_{n}=B_{n} \sum_{k=1}^{n}\left(X_{k}-b_{n}\right): n \in \mathbb{N}\right\}$, and let $\phi: \mathbb{N} \rightarrow D$ be given by $\phi(n)=(1 / n, \psi(n))$ using (3.3). Then for $\Delta=\{0\} \times[1, c]=\bar{D} \backslash D$ and any sequence $n_{k} \in \mathbb{N}$ with $\phi\left(n_{k}\right) \rightarrow(0, t) \in \Delta$ we have $n_{k} \rightarrow \infty$ and $\psi\left(n_{k}\right) \rightarrow t$ so that we get

$$
Y_{n_{k}}=B_{n_{k}} \sum_{\ell=1}^{n_{k}}\left(X_{\ell}-b_{n_{k}}\right) \Rightarrow \mu_{t}=t^{-E}\left(\mu^{t} * \delta_{-a(t)}\right)
$$

as described above. Hence (2.1) is fulfilled for the weakly continuous family of distributions $\left\{\mu_{0, t}=\mu_{t}=t^{-E}\left(\mu^{t} * \delta_{-a(t)}\right):(0, t) \in \Delta\right\}$, where $\mu_{1}=\mu=\mu_{c}$. Further the assumptions of Corollary 3 imply $\phi\left(T_{n}\right) \Rightarrow \delta_{0} \otimes \rho$ and hence by Theorem 1 the assertion follows. Corollary 3 is proved.

As an example, it is implicitly shown in [5] that for any $c>1$ the logarithmic summation with

$$
\mathbb{P}\left\{T_{n}=k\right\}=\left\{\begin{array}{ll}
D_{n}^{-1} k^{-1} & \text { if } 1 \leqslant k \leqslant n, \\
0 & \text { else, }
\end{array} \text { with } \quad D_{n}=\sum_{k=1}^{n} \frac{1}{k} \sim \ln n\right.
$$

fulfills $\psi\left(T_{n}\right) \Rightarrow \rho$ for the logarithmic distribution $\rho$ on $[1, c]$ with probability density $t \mapsto$ $(t \ln c)^{-1} 1_{[1, c]}(t)$. Hence by Corollary 3 we get for the distributions $\nu_{k}$ of $B_{k} \sum_{\ell=1}^{k}\left(X_{\ell}-b_{k}\right)$

$$
\frac{1}{\ln n} \sum_{k=1}^{n} \frac{1}{k} \nu_{k} \rightarrow \frac{1}{\ln c} \int_{1}^{c} t^{-E}\left(\mu^{t} * \delta_{-a(t)}\right) \frac{\mathrm{d} t}{t} \quad \text { weakly as } n \rightarrow \infty .
$$

Further examples of random variables $T_{n}$ (corresponding to summability methods) with $\psi\left(T_{n}\right)$ converging to the logarithmic distribution are given in [5].

3.3. Transfer theorems for random allocations. Let $n$ balls be allocated to $N$ boxes independently and with equal probability. Denote by $\mu_{r}(n, N)$ the final number of boxes containing exactly $r \geqslant 0$ balls, and let

$$
\mu_{r}^{*}(n, N)=\frac{\mu_{r}(n, N)-\mathbb{E}\left[\mu_{r}(n, N)\right]}{\sqrt{\mathbb{D}^{2}\left[\mu_{r}(n, N)\right]}}, \quad 0 \leqslant r \leqslant n,
$$

denote the standardized random variables. The limit behavior of $\mu_{r}^{*}(n, N)$ as simultaneously $n, N \rightarrow \infty$ is well known. The possible limit distributions are either the standard 
normal distribution $\mathscr{N}_{0,1}$ or a standardized Poisson distribution $\pi_{\lambda}^{*}$ with parameter $\lambda>0$. For details we refer to the monograph [24] and the literature cited therein. For random numbers of balls and boxes we can derive the following transfer theorems as applications of Theorem 1. Let $\left\{\left(T_{n}, U_{N}\right): n, N \in \mathbb{N}\right\}$ be random variables in $\mathbb{N}^{2}$ such that $\left(T_{n}, U_{N}\right)$ is independent of the allocations and thus of $\mathscr{F}_{r}=\left\{\mu_{r}^{*}(n, N): n, N \in \mathbb{N}, n \geqslant r\right\}$ for every $(n, N) \in \mathbb{N}^{2}$ and every $r \geqslant 0$. The following transfer theorem for the number of empty boxes $\mu_{0}(n, N)$ is already known by [4]. Its method of proof inspired our general version, but the proof of Theorem 1 is even simpler than the proof given for the special result in [4]. In turn Theorem 1 can be applied to derive transfer theorems for $\mu_{r}(n, N)$ with $r \geqslant 1$. Partial results can already be found in [21].

Corollary 4. Let $\Delta_{0}=\left(\mathbb{R}_{+} \times\{0\}\right) \cup\left(\{0\} \times \mathbb{R}_{+}\right)$, and for any $(g, d) \in \Delta_{0}$ let

$$
\nu_{g, d}= \begin{cases}\pi_{1 / g}^{*} & \text { if } 0<g<\infty, d=0, \\ \mathscr{N}_{0,1} & \text { if } g=d=0, \\ \pi_{1 / d}^{*} & \text { if } g=0,0<d<\infty\end{cases}
$$

which defines a weakly continuous family $\left\{\nu_{g, d}:(g, d) \in \Delta_{0}\right\}$ of probability distributions. Assume that for some probability distribution $\rho_{0}$ on $\Delta_{0}$

$$
\phi_{0}\left(T_{n}, U_{N}\right)=\left(\frac{2 U_{N}}{T_{n}^{2}}, \frac{e^{T_{n} / U_{N}}}{U_{N}}\right) \Rightarrow \rho_{0} .
$$

Then we have

$$
\mu_{0}^{*}\left(T_{n}, U_{N}\right) \Rightarrow \int_{\Delta_{0}} \nu_{g, d} \mathrm{~d} \rho_{0}(g, d)
$$

Corollary 5. Let $\Delta_{1}=\{0\} \times \mathbb{R}_{+}$, and for any $(0, d) \in \Delta_{1}$ let

$$
\nu_{d}= \begin{cases}\mathscr{N}_{0,1} & \text { if } d=0, \\ \pi_{1 / d}^{*} & \text { if } 0<d<\infty,\end{cases}
$$

which defines a weakly continuous family $\left\{\nu_{d}:(0, d) \in \Delta_{1}\right\}$ of probability distributions. Assume that for some probability distribution $\rho_{1}$ on $\mathbb{R}_{+}$

$$
\phi_{1}\left(T_{n}, U_{N}\right)=\left(\frac{U_{N}}{T_{n}^{2}}, \frac{e^{T_{n} / U_{N}}}{T_{n}}\right) \Rightarrow \delta_{0} \otimes \rho_{1} .
$$

Then we have

$$
\mu_{1}^{*}\left(T_{n}, U_{N}\right) \Rightarrow \int_{0}^{\infty} \nu_{d} \mathrm{~d} \rho_{1}(d)
$$

Corollary 6. For $r \geqslant 2$ let $\Delta_{r}=\{0\} \times \mathbb{R}_{+}$. For any $(0, d) \in \Delta_{r}$ again let

$$
\nu_{d}= \begin{cases}\mathscr{N}_{0,1} & \text { if } d=0, \\ \pi_{1 / d}^{*} & \text { if } 0<d<\infty .\end{cases}
$$

Assume that for some probability distribution $\rho_{r}$ on $\mathbb{R}_{+}$

$$
\phi_{r}\left(T_{n}, U_{N}\right)=\left(\frac{1}{T_{n}}, \frac{r ! U_{N}^{r-1}}{T_{n}^{r}} e^{T_{n} / U_{N}}\right) \Rightarrow \delta_{0} \otimes \rho_{r} .
$$

Then we have

$$
\mu_{r}^{*}\left(T_{n}, U_{N}\right) \Rightarrow \int_{0}^{\infty} \nu_{d} \mathrm{~d} \rho_{r}(d)
$$

P r o o f of Corollaries 4-6. For any sequences $\left(n_{k}\right)_{k \in \mathbb{N}}$ and $\left(N_{k}\right)_{k \in \mathbb{N}}$ of positive integers such that $\phi_{r}\left(n_{k}, N_{k}\right) \rightarrow(g, d) \in \Delta_{r}$ for some $r \geqslant 0$, it can easily be shown that $n_{k} \rightarrow \infty, N_{k} \rightarrow \infty$ and that $\mu_{r}^{*}\left(n_{k}, N_{k}\right)$ converges in distribution to the corresponding normal or Poissonian distribution given in Corollaries 4-6. For $r=0$ this is already shown in the proof of Theorem 2.1 in [4] and for $r \geqslant 1$ it follows in the same spirit using the limit 
theorems given in [24]. Hence for an application of Theorem 1 we simply have to argue that $\phi_{r}: \mathbb{N}^{2} \rightarrow D=\operatorname{Im} \phi_{r}$ is a homeomorphism for any $r \geqslant 0$. Again, for $r=0$ these arguments have been given in the proof of Theorem 2.1 in [4] and for $r \geqslant 1$ they can also be derived easily.

\section{REFERENCES}

1. Aksomaitis A On convergence of the maximum of dependent random variables. Lith. Math. J., 1992, v. 32, № 1, p. 1-3.

2. Aksomaitis A. Rate of convergence in the limit transfer theorem of the maximum and minimum. - Proceedings of the Sixth Vilnius Conference on Probability Theory and Mathematical Statistics. Ed. by B. Grigelionis. Utrecht: VSP, 1994, p. 35-42.

3. Becker-Kern P. Stable and semistable hemigroups: Domains of attraction and selfdecomposability. - J. Theoret. Probab., 2003, v. 16, № 3, p. 573-598.

4. Becker-Kern P. An almost sure limit theorem for mixtures of domains in random allocation. - Studia Sci. Math. Hungar., 2007, v. 44, № 3, p. 331-354.

5. Becker-Kern P. Almost sure limit theorems of mantissa type for semistable domains of attraction. - Acta Math. Hungar., 2007, v. 114, № 4, p. 301-336.

6. Becker-Kern P., Meerschaert M. M., Scheffler H. P. Limit theorem for continuous-time random walks with two time scales. - J. Appl. Probab., 2004, v. 41, № 2, p. 455-466.

7. Bening V. E., Korolev V. Yu. Generalized Poisson Models and their Applications in Insurance and Finance. Utrecht: VSP, 2002, $434 \mathrm{p}$.

8. Berman S. M. Limiting distribution of the maximum term in sequences of dependent random variables. - Ann. Math. Statist., 1962, v. 33, p. 894-908.

9. Биллингсли П. Сходимость вероятностных мер. М.: Наука, 1977, 351 с.

10. Csörgö S., Megyesi Z. Merging to semistable laws. - Теория вероятн. и ее примен., 2002 , т. 47 , в. 1 , с. $90-109$.

11. Dion J. P., Yanev N. M. Limit theorems and estimation theory for branching processes with an increasing random number of ancestors. - J. Appl. Probab., 1997, v. 34, № 2, p. 309-327.

12. Dudley R. M. Real Analysis and Probability. Cambridge: Cambridge Univ. Press, 2002, 555 p.

13. Finkelstein M., Kruglov V. M., Tucker H. G. Convergence in law of random sums with non-random centering. - J. Theoret. Probab., 1994, v. 7, № 3, p. 565-598.

14. Gnedenko B. V. On limit theorems for a random number of random variables. Lecture Notes in Math., 1983, v. 1021, p. 167-176.

15. Гнеденко Б. В., Фахим Х. Об одной теореме переноса. - Докл. АН СССР, 1969, т. 187 , № 1 , c. $15-17$.

16. Gnedenko B. V., Korolev V. Yu. Random Summation. Boca Raton: CRC Press, 1996, $267 \mathrm{p}$.

17. Gut A. Stopped Random Walks. New York: Springer, 1988, 199 p.

18. Хацод $B$. О предельном поведении произведений случайного числа независимых одинаково распределенных случайных величин со значениями в группе. - Теория вероятн. и ее примен., 1994, т. 39, в. 2, с. 374-394.

19. Hazod W., Siebert E. Stable Probability Measures on Euclidean Spaces and on Locally Compact Groups. Structural Properties and Limit Theorems. Dordrecht: Kluwer, 2001, 612 p.

20. Heyer H. Structural Aspects in the Theory of Probability. Hackensack: World Scientific, 2010, $412 \mathrm{p}$.

21. Ивченко Г. И., Медведев Ю. И., Севастьянов Б. А. Размещение случайного числа частиц по ячейкам. - Матем. заметки, 1967, т. 1, в. 5, с. 549-554.

22. Joag-Dev K., Proschan F. Negative association of random variables, with applications. - Ann. Statist., 1983, v. 11, p. 286-295.

23. Kallenberg $O$. Foundations of Modern Probability. New York: Springer, 2002, 638 p.

24. Колчин В.Ф., Севастьянов Б.А., Чистяков В. П. Случайные размещения. М.: Наука, 1976, 223 с. 
25. Korolev $V$. Y., Kossova E. $V$. On limit distributions of randomly indexed multidimensional random sequences with operator normalization. - J. Math. Sci. (N.Y.), 1994, v. 72, № 1, p. 2915-2929.

26. Korolev V. Y., Kossova E. V. Convergence of multidimensional random sequences with independent random indices. - J. Math. Sci. (N.Y.), v. 76, № 2, p. 2259-2268.

27. Korolev V. Y., Kruglov V. M. Limit theorems for random sums of independent random variables. - Lecture Notes in Math., 1993, v. 1546, p. 100-120.

28. Kowalski P., Rychlik $Z$. On the products of a random number of independent random variables. - Bull. Pol. Acad. Sci. Math., 1995, v. 43, № 3, p. 219-230.

29. Meerschaert M. M., Scheffler H. P. Limit Distributions for Sums of Independent Random Vectors. Heavy Tails in Theory and Practice. Chichester: Wiley, 2001, 484 p.

30. Parthasarathy K. R. Probability Measures on Metric Spaces. London: Academic Press, 1967, 276 p.

31. Robbins $H$. The asymptotic distribution of the sum of a random number of random variables. - Bull. Amer. Math. Soc., 1948, v. 54, p. 1151-1161.

32. Siegel $G$. Convergence of randomly selected sums in a separable Banach space. Math. Nachr., 1988, v. 139, p. 139-153.

33. Silvestrov D. S. Limit Theorems for Randomly Stopped Stochastic Processes. London: Springer, 2004, $398 \mathrm{p}$.

34. Thomas D.I. On limiting distributions of a random number of dependent random variables. - Ann. Math. Statist., 1972, v. 43, p. 1719-1726.

35. Zhang L. X., Wen J. A weak convergence for negatively associated fields. - Statist. Probab. Lett., 2001, v. 53, № 3, p. 259-267. 